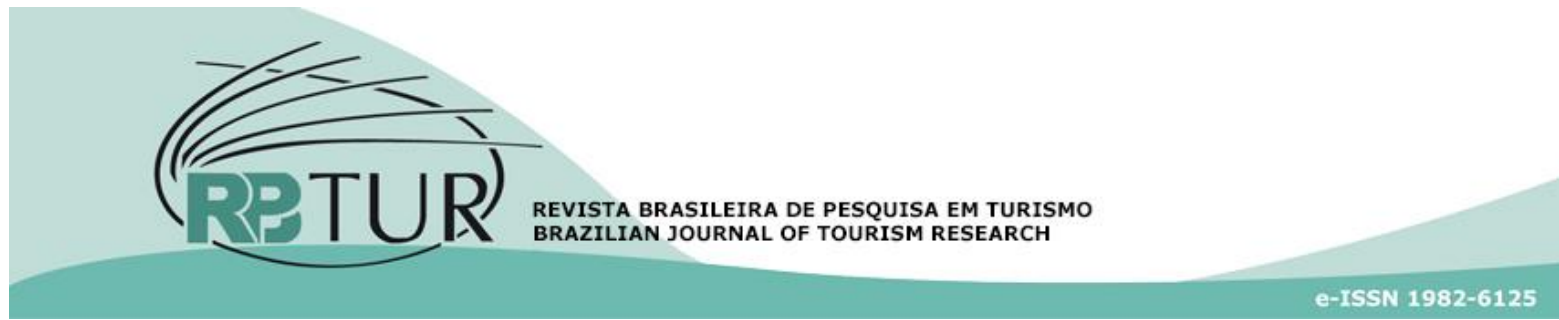

\title{
Artigos
}

\section{A influência da percepção de utilidade de avaliações online na intenção de compra de serviços de hotelaria}

\section{The effect of perceiveid usefulness of online reviews on hotel booking intentions}

\section{La influencia de la percepción de utilidade de evaluaciones online en la intención de compra de servicios de hotelería}

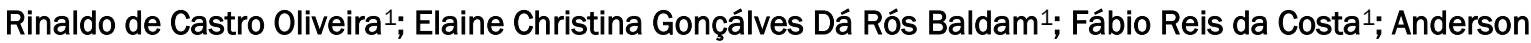 \\ Soncini Pelissari ${ }^{1}$
}

1 Universidade Federal de Espirito Santo (UFES), Vitória, ES, Brasil.

Palavras-chave:

Serviços de hotelaria;

Avaliações online de consumidores; Percepção de utilidade da informação;

Intenção de compra.
Resumo

O crescimento da internet facilitou a interconectividade dos consumidores por meio de fó runs online que permitem os consumidores a gerar conteúdo e influenciar os outros em suas decisões de compra. 0 objetivo desta pesquisa foi identificar a influência da percepção de utilidade das avaliações online na intenção de compra de serviços de hotelaria. Utilizou-se de abordagem quantitativa, onde foram aplicados questionários junto a consumidores que usam avaliações online para reserva de hospedagens, e os dados foram analisados por meio de modelagem de equações estruturais. Os resultados mostraram a influência direta da percepção de utilidade da informação na intenção de compra, sendo que os construtos antecessores, descritos como necessidade da informação, credibilidade da informação e qualidade da informação, apresentaram impacto positivo e significativo na percepção de utilidade das avaliações online. Comparando esses resultados com pesquisa realizada por Erkan e Evans (2016) com consumidores do Reino Unido que se utilizam de midas sociais para decisão sobre suas compras, neste estudo a credibilidade da informação mostrou-se mais relevante em relação à qualidade da informação, evidenciando um comportamento mais cético dos consumidores brasileiros. Os resultados encontrados merecem atenção dos profissionais que gerenciam o marketing digital das organizações inseridas nesse ambiente, principalmente em relação a importância da gestão da credibilidade e da qualidade das avaliações online na intenção de compra de serviços de hotelaria, sendo esta uma contribuição prática resultante da pesquisa.

Abstract

The growth of the Internet has enabled consumer-to-consumer interactions through online platforms where users share content and influence the purchase decisions of other consumers. The objective of this research is to identify the effect of perceived usefulness of online reviews on hotel booking intentions. The approach is quantitative, using a questionnaire to collect data from consumers who use online reviews before booking a hotel. The data were analyzed using structural equation modeling. The results showed the direct influence of perceived information usefulness on purchase intention, and the antecedent constructs- 
Palabras clave:

Servicios de hoteleira;

Evaluaciones online de consumidores;

Percepción de utilidad de la información;

Intención de compra.

Revisado por pares.

Recebido em: 01/04/2019.

Aprovado em: 22/05/2019.
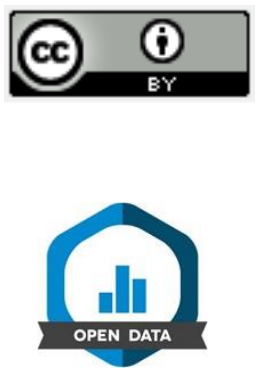

needs of information, information credibility, and information quality-had a positive and sig nificant impact on perceived usefulness of online reviews. Comparing these results with research by Erkan and Evans (2016) conducted with UK consumers that use social media to decide about their purchases, in this study information credibility was more relevant than information quality, suggesting a more skeptical behavior of Brazilian consumers. These findings have implications for practitioners that manage the digital marketing of organizations inserted in this environment, mainly regarding the impact of credibility and quality of online reviews on hotel booking intentions, being this a practical contribution of the research.

\section{Resumen}

El crecimiento del Internet ha facilitado la interconectividad de los consumidores a través de foros en línea que permiten a los consumidores generar contenido e influir a otros en sus decisiones de compra. El objetivo de esta investigación fue identificar la influencia de la percepción de la utilidad de las evaluaciones online en la intención de compra de servicios de hotelería. Se utilizó un enfoque cuantitativo, donde se aplicaron cuestionarios a consumidores que utilizan evaluaciones en línea para la reserva de hospedaje. Los datos se analizaron mediante el modelado de ecuaciones estructurales. Los resultados mostraron la influencia directa de la percepción de la utilidad de la información en la intención de compra, siendo que los constructos predecesores, descritos como necesidad de la información, credibilidad de la información y calidad de la información, presentaron impacto positivo y significativo en la percepción de utilidad de las evaluaciones online. Comparando estos resultados con una investigación realizada por Erkan e Evans (2016) con consumidores del Reino Unido que utilizan las redes sociales para decidir sobre sus compras, en este estudio la credibilidad de la información se mostró más relevante en relación a la calidad de la información, evidenciando un comportamiento más escéptico de los consumidores brasileños. Los resultados encontrados merecen la atención de los profesionales que administran el marketing digital de las organizaciones insertadas en ese ambiente, principalmente en relación con la importancia de la gestión de la credibilidad y de la calidad de las evaluaciones online en la intención de compra de servicios de hoteleira, siendo esta una contribución práctica resultante de la investigación.

Como citar: Oliveira, R. C.; Baldam, E. C. G. R.; Costa, F. R.; Pelissari, A. S. (2020). A influência da percepção de utilidade de avaliações online na intenção de compra de serviços de hotelaria. Revista Brasileira de Pesquisa em Turismo, São Paulo, 14 (2), p. 30-45, maio/ago. http://dx.doi.org/10.7784/rbtur.v14i2.1695

Dados abertos: http://dx.doi.org/10.17632/n8ctb4fcg5.1

\section{INTRODUÇÃO}

A Internet tem proporcionado grandes oportunidades para os consumidores discutirem suas opiniões com os outros. No início da era da Internet, fóruns de discussão online encorajavam os usuários a falar sobre diversos assuntos, porém, com o tempo, esses fóruns tornaram-se cada vez mais especializados, centrados em discussões de tópicos específicos (Erkan \& Evans, 2016).

Almeida (2018) esclarece que até o início do século XXI a forma mais conhecida para verificar a qualidade do serviço e sua contribuição para a satisfação tenha consistido na realização de surveys, entretanto a expansão da internet mudou este cenário, pois a popularização da internet possibilitou a criação de diferentes redes sociais nas quais as pessoas expõem diversos aspectos de suas vidas. Nos últimos anos, um crescente número de plataformas de opinião que oferecem aos consumidores avaliações online e classificações de produtos têm sido criados (Ladhari \& Michaud, 2015).

Dentro desse novo ambiente de troca de informações, o mercado de reservas online de serviços de hotelaria vem crescendo, atingindo nos EUA receita de 1,48 bilhões de dólares em 2016, tendo como maiores sites o TripAdvisor, Hotels.com, Marriott International, Booking.com e Airbnb. Juntas, essas empresas representaram $28,1 \%$ de participação no mercado norte-americano em 2016, ano em que as vendas de viagens online globais totalizaram 564,87 bilhões de dólares, com projeções de crescimento para 755,94 bilhões de dólares em 2019 (Statista.Com, 2018).

De acordo com pesquisa realizada na América Latina, o Latin America Online Travel Overview, publicado pela Phocuswright.com, o mercado total de viagens online da América Latina atingiu US $\$ 60,2$ bilhões em 2015 , sendo que Brasil e México representam mais de 70\% deste mercado. 0 estudo aponta ainda que as viagens online crescerão em média 10\% nos próximos anos na América Latina (PHOCUSWRIGHT.COM, 2016). Esses números reforçam a relevância dessa nova modalidade de compra de serviços associados ao mercado de viagens e turismo brasileiro, bem como o seu potencial de crescimento. Ainda segundo o site de pesquisa 
Phocuswright (2018), conforme apresentado em seu relatório intitulado The Brazilian Digital Traveler, apesar da recente incerteza econômica e política, o mercado de viagens online do Brasil tem sido resiliente e continua a crescer, devido aos consumidores adeptos à tecnologia, e por isso, as empresas de viagens precisam entender os comportamentos e preferências do consumidor que influenciam as decisões de compra.

É importante destacar que os serviços de hospedagem se encontram no último elo da cadeia dos serviços turísticos e configuram-se como um dos mais relevantes, representando a base de permanência temporária do turista, que busca encontrar uma extensão de sua residência (IBGE, 2016). Colaborando com essa visão, Medeiros, Gosling e Vera (2015) observam que tais serviços são os principais responsáveis por sentimentos de insatisfação dos consumidores durante suas viagens. Assim, antes da consolidação de uma reserva de hospedagem, existem diversos fatores que influenciam os consumidores, como as experiências já vivenciadas, as informações adquiridas, as quais com o passar do tempo vem se tornando cada vez mais sofisticadas (Silva, Mendes Filho, \& Marques Júnior, 2019).

Sabe-se que a satisfação tem desempenhado um papel cada vez mais importante para a manutenção da competitividade de hotéis e pousadas, principalmente por causa da variedade de canais disponíveis para que os consumidores divulguem suas experiências com os meios de hospedagem, tanto as boas, quanto as ruins (Almeida, 2018). Porém, muitos hotéis não sabem ao certo como responder às opiniões de usuários, e esta incerteza pode, em parte, ser devido à falta de compreensão de como os consumidores usam as opiniões online para compra de produtos e serviços (Ong, 2012). Diante desse contexto, é cada vez mais importante que os gestores de serviços de hotelaria reconheçam a influência desse novo canal de comunicação em seus negócios, para desenvolverem ações gerenciais que melhorem a qualidade do serviço, e como forma de entenderem o impacto das opiniões de usuários no processo de escolha de clientes potenciais.

Os estudos elaborados por Browning, So e Sparks (2013), Hernández-Méndez, Muñoz-Leiva, SánchezFernández (2015), Ladhari e Michaud (2015), Ong (2012), Park, Xiang, Josiam e Kim (2014) e Tsao, Hsieh, Hsieh e Lin (2015) avaliaram a influência das opiniões de consumidores online de serviços hoteleiros, porém ainda são restritas e merecem aprofundamentos, especialmente por não abordarem o efeito da percepção de utilidade das avaliações online na intenção de compra de serviços de hotelaria, sendo esta a principal lacuna abordada nesta pesquisa. Considera-se também, o fato destes serviços apresentarem alto grau de subjetividade, por sua natureza mais intangível, além de serem susceptíveis a fatores socioculturais. Com isso, o objetivo central desta pesquisa foi identificar a influência da percepção de utilidade das avaliações online na intenção de compra de serviços de hotelaria no Brasil. Como objetivo específico, a pesquisa se propôs também a avaliar as variáveis antecessoras que impactam a percepção de utilidade das avaliações online.

Avanços recentes na internet e o desenvolvimento de mídias sociais facilitaram a interconectividade dos consumidores, por meio de fóruns online, comunidades virtuais, que fornecem avaliações e recomendações, gerando um novo fluxo no comércio eletrônico, que permite aos consumidores gerar conteúdo e influenciar outros, num processo de cocriação de valor (Hajli, 2013). Reconhecer os fatores que influenciam a percepção de utilidade das informações produzidas e difundidas nas novas mídias digitais, bem como o efeito desta percepção na intenção de compra dos consumidores de serviços de hotelaria, caracteriza-se como a principal contribuição teórica deste estudo, com implicações práticas na gestão destes serviços. Por isso, a pesquisa se justifica por abordar um tema novo e relevante, que tem cada vez mais impactado os processos de marketing e de vendas de serviços de hospedagem.

\section{ARCABOUÇO TEÓRICO DE REFERÊNCIA}

O modelo proposto neste estudo é uma adaptação do modelo de aceitação da informação desenvolvido por Erkan e Evans (2016). Este modelo é o resultado da ampliação do modelo de adoção da informação desenvolvido por Sussman et al., (2003), a partir da introdução de componentes relacionados à teoria da ação racional de Fishbein e Ajzen (1975).

Segundo Erkan e Evans (2016), o modelo de aceitação da informação mostra que a influência das avalições online disponíveis nas mídias sociais não depende apenas das características das informações online, como 
qualidade e credibilidade, mas também depende do comportamento dos consumidores em relação a essas informações. Portanto, a revisão de literatura elaborada para embasar teoricamente a presente pesquisa tem foco no modelo de aceitação da informação, com ênfase nos construtos que compõem a percepção de utilidade da informação, e influenciam o processo de intenção de compra.

Estudos recentes têm buscado entender a influência das avaliações online, disponibilizadas em mídias sociais e sites especializados, na intenção de compra de consumidores de produtos e serviços, como por exemplo, Erkan e Evans (2016), Wani, Ali e Farooq (2016), Elseidi e El-Baz (2016), Farley e Murched (2016), Bataineh, (2015), Haji (2013) e Jalilvand e Samiei (2012). Ao tomar sua decisão, os consumidores muitas vezes obtêm recomendações de amigos por meio de boca-a-boca, se referenciam com base em informações disponibilizadas nas mídias, incluindo campanhas publicitárias e de marketing, ou consultam fontes na internet (Browning, So \& Sparks, 2013).

Para Jardim e Sant'Anna (2007), com a revolução digital, marcada por transformações velozes nas tecnologias da informação e de comunicação impulsionadas pela internet, o setor do turismo tem se inserido com rapidez no mundo online com a intenção de vender e divulgar seus negócios. Portanto, a internet está sendo usada cada vez mais pelos consumidores para embasar suas decisões sobre qual destino de férias visitar ou hotel reservar, pois sem realmente experimentar o hotel ou o destino de férias, os viajantes têm oportunidades limitadas de avaliar a qualidade do serviço que receberão, e se atenderão às suas expectativas (Browning, So \& Sparks, 2013).

Se os consumidores levarem em consideração as mensagens online, elas poderão se transformar imediatamente em uma ação de compra por meio dos sites das empresas ou de compras, tornando as avaliações online muito poderosas, o que leva os pesquisadores a se interessarem em como esse processo influencia a intenção de compra (Erkan \& Evans, 2016). Com isso, é importante reconhecer qual é a percepção de utilidade que os consumidores possuem em relação às opiniões disponibilizadas nas plataformas online de reserva de serviços de hospedagem, e como essa informação afeta esse processo de decisão.

A utilidade da informação refere-se à percepção das pessoas de que o uso de novas informações melhorará seu desempenho, levando-as a perceber que essas opiniões poderiam ser úteis para ajudá-las a melhorar suas decisões de compra (Cheung, Lee \& Rabjohn, 2008), e é considerada como a preditora principal da adoção da informação (Davis, 1989; Erkan \& Evans, 2016) e da intenção de compra (Lee \& Koo, 2015) porque os consumidores tendem a se envolver com as informações quando a acham útil.

Erkan e Evans (2016) consideraram em seu modelo de aceitação da informação que a percepção de utilidade da informação tem como variável antecessora a qualidade da informação. A qualidade das avaliações online pode ser descrita como o poder de persuasão de comentários expressos numa mensagem informativa (Bhattacherjee \& Sanford, 2006). Quando os clientes buscam informações, a qualidade da informação possivelmente terá impacto na aceitação do cliente em relação aos canais de comunicação que disponibilizam as avaliações online (Cheung \& Thadani, 2012).

Park, Lee e Han (2007) definiram a qualidade das avaliações online como a qualidade do seu conteúdo na perspectiva das características da informação, ou seja, quanto à relevância, compreensibilidade, suficiência e objetividade. Pesquisas anteriores descobriram que a qualidade das avaliações online influencia positivamente a intenção de compra (Bataineh, 2015; Erkan \& Evans, 2016; Lee \& Shin, 2014; Park, Lee \& Han, 2007). Assim, a presente pesquisa se propõe a avaliar o efeito da qualidade das avaliações online na percepção de utilidade da informação, e, por consequência, na intenção de compra de clientes, tendo como hipótese a seguinte proposição.

H1: A qualidade das avaliações online influencia positivamente a percepção de utilidade da informação.

Ao levar em consideração a enorme quantidade de informação que os indivíduos compartilham online, a maioria deles pode precisar, durante as compras, de uma referência para fortalecer sua confiança, visando diminuir a sensação de cometer erros ou riscos, porque a quantidade de comentários online pode ser um sinal de quanto o produto é valioso e popular (Bataineh, 2015). Segundo Lee, Park e Han (2008) e Bataineh (2015), a quantidade de informações recebidas pelos clientes afeta as decisões dos mesmos em adquirir produtos e serviços. 
Park, Lee e Han (2007) mediram a quantidade de opiniões pelo número de avaliações de consumidores online de um produto, e avaliaram que a quantidade de avaliações online provavelmente indicava ao leitor a popularidade do produto e, possivelmente, o sucesso de vendas. Além disso, muitas avaliações de usuários provavelmente levariam os consumidores a racionalizar suas decisões de compra por associar essa elevada quantidade de opiniões a uma provável qualidade do produto, podendo servir como estratégia de redução de risco e, por sua vez, levar a maiores intenções de compra (Park, Lee \& Han, 2007). Dessa forma, a presente pesquisa se propõe a avaliar o efeito da quantidade de avaliações online na percepção de utilidade da informação, tendo como hipótese a seguinte proposição.

H2: A quantidade das avaliações online influencia positivamente a percepção de utilidade da informação.

A necessidade de informação é outro fator determinante no processo de intenção de compra dos consumidores (Erkan \& Evans, 2016). Wolny e Mueller (2013) consideram-na como um dos motivadores das opiniões online. Dada a crescente disponibilidade e popularidade das plataformas web de opinião de usuários, as avaliações online têm se tornado um fenômeno proeminente e têm aumentado sua importante função na decisão de compra dos consumidores (Browning, So \& Sparks, 2013; Zhu \& Zhang, 2010). Conforme Ladhari e Michaud (2015), as mídias sociais fornecem uma plataforma para busca de informação de uso fácil e amigável, e tem se tornado indispensável. Neste estudo, conforme adotado por Erkan e Evans (2016), a necessidade de informações foi considerada como um fator antecedente que influencia a percepção de utilidade da informação. Dessa forma, como hipótese a ser testada, apresenta-se a seguinte proposição.

H3: A necessidade das avaliações online influencia positivamente a percepção de utilidade da informação.

A credibilidade da informação refere-se às percepções dos destinatários da mensagem sobre a sua veracidade (Erkan \& Evans, 2016). Segundo Ladhari e Michaudi (2015), as opiniões online são vistas como uma fonte de informação confiável e imparcial. Entretanto, dada a importância das avaliações de turistas como fontes de informações relacionadas a viagens, a credibilidade da fonte está se tornando uma questão cada vez mais crucial em termos de pesquisa e aplicação, visto que há preocupações sobre a falta de credibilidade das avaliações online de viagens (Park, et al., 2014). Quanto maior for a sensação de risco no ato de se escolher uma hospedagem, maior será a procura do cliente por conhecimento sobre o produto de seu interesse, principalmente se a compra for feita por meio do comércio eletrônico (Jardim \& Sant'Anna, 2007).

Park et al. (2014) demonstraram que a exibição de informações pessoais, como interesse e localização da viagem, pode facilitar a avaliação da credibilidade e relevância das avaliações online, o que sugere que a confiança e a credibilidade podem ser fomentadas entre os pares que compartilham informações sobre tópicos de interesse. Os resultados do estudo de Erkan e Evans (2016) mostraram o efeito positivo direto da credibilidade da informação sobre a utilidade da informação, concluindo que os consumidores consideram a informação nas redes sociais útil, quando a mesma é crível. Com isso, a seguinte hipótese foi postulada:

H4: A credibilidade das avaliações online influencia positivamente a percepção de utilidade da informação.

Erkan e Evans (2016) constataram uma influência positiva da percepção de utilidade da informação sobre a intenção de compra. Além disso, Lee e Koo (2015) realizaram um experimento com avaliações online para testar a relação entre a utilidade da mensagem e a intenção de compra, onde os resultados mostraram que a utilidade da informação está positivamente associada à intenção de compra. Assim, a seguinte hipótese foi postulada.

H5: A percepção de utilidade das avaliações online influencia positivamente a intenção de compra.

Considerando os conceitos teóricos apresentados, e as referências de estudos empíricos anteriores que embasam as hipóteses postuladas, desenvolveu-se o modelo estrutural tendo como base o estudo de Erkan e Evans (2016). 0 modelo de aceitação da informação proposto por estes autores (2016) examina as relações entre os seguintes construtos: qualidade da informação, credibilidade da informação, necessidades de informação, atitude em relação à informação, utilidade da informação, adoção da informação e intenção de compra. De acordo com os resultados encontrados por Erkan e Evans (2016), a adoção da informação não se mostrou estatisticamente significativa em relação à percepção de utilidade da informação, e por essa 
razão, nesse estudo optou-se por não avaliar esse construto no modelo estrutural. A variável quantidade de informação considerada no modelo desenvolvido por Bataineh (2015) foi adicionada ao modelo adaptado de Erkan e Evans (2016), visando possibilitar a análise do efeito do número de avaliações online na percepção de utilidade da informação. Dessa forma, a presente pesquisa visa identificar as relações entre os construtos apresentados na Figura 1.

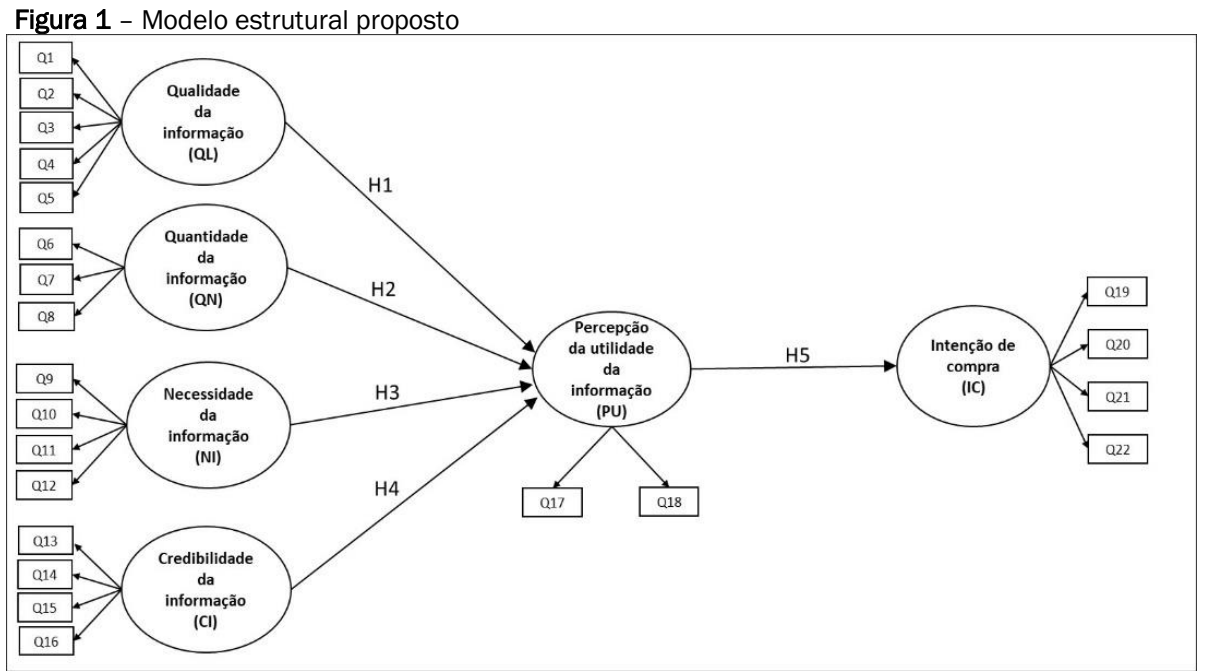

Fonte: Elaborado pelos autores

\section{ASPECTOS METODOLÓGICOS DA INVESTIGAÇÃO}

A pesquisa proposta é empírica e de natureza descritiva-quantitativa, uma vez que procurou identificar a influência das opiniões dos usuários de serviços de hotelaria na intenção de compra futura de clientes potenciais desses serviços, por meio de pesquisa utilizando-se questionário fechado para coleta dos dados, e instrumentos estatísticos para tratamento dos mesmos, visando medir as relações entre as variáveis estabelecidas no modelo estrutural.

A coleta de dados foi feita em julho de 2018 utilizando-se questionário eletrônico, com escala do tipo Likert de cinco pontos. Devido à maior facilidade de acesso, a amostra foi composta por alunos, professores e funcionários de instituições de ensino localizadas no estado do Espírito Santo. 0 questionário foi elaborado a partir da revisão da literatura, contemplando os construtos e variáveis a serem investigadas. Foram utilizadas como referências as escalas de mensuração adotadas nos estudos de Erkan e Evans (2016) e Bataineh (2015), adaptando-se o questionário para o contexto dos serviços de hotelaria, com o intuito de atender os objetivos da presente pesquisa. Os indicadores e as escalas de mensuração, com as respectivas referências, são apresentados no Apêndice A.

Como perguntas de controle, visando selecionar respondentes com perfil adequado para participação da pesquisa, utilizou-se como referência as mesmas questões adotadas no estudo de Ong (2012). Em síntese, as perguntas procuravam avaliar o grau de utilização das avaliações online para reserva de hospedagem, e a frequência que os respondentes acessaram avaliações online para escolher uma hospedagem nos últimos doze meses. Em relação à primeira questão, os respondentes que não usam avaliações online, e/ou que nunca reservaram uma hospedagem, foram excluídos da base de dados. Em relação à segunda questão, para participarem da pesquisa, os respondentes deveriam ter reservado pelo menos uma hospedagem nos últimos doze meses, utilizando-se de opiniões online.

Para verificação da aderência do questionário adaptado à realidade do mercado brasileiro, foi realizado um pré-teste com 37 pessoas selecionadas por conveniência. A partir dos resultados estatísticos obtidos, pequenos ajustes foram feitos no instrumento de coleta, visando facilitar o entendimento das questões. Após a coleta dos dados, a análise dos resultados foi feita utilizando-se de metodologia estatística multivariada, mais especificamente a Modelagem de Equações Estruturais (MME), a partir do uso do software RStudio, versão 1.1.453, por ser este um software de código aberto que permite o uso de múltiplos algoritmos. Especificamente nesta pesquisa utilizou-se o algoritmo PLS-SEM. Diante dos resultados 
encontrados, foram elaboradas as análises e interpretações dos mesmos, visando avaliar as hipóteses estabelecidas no referencial teórico do estudo.

\subsection{Amostra e tratamento dos dados}

No presente estudo foram coletadas 252 respostas ao todo, e os dados foram submetidos à análise quanto ao atendimento das perguntas de controle, a ocorrência de dados ausentes e a observação de respostas com comportamento suspeito. Do total de respostas, 53 não atenderam os critérios estabelecidos nas perguntas de controle, e, portanto, foram excluídos da base de dados. Em dois casos verificou-se respostas com notas iguais para todas as questões, o que caracteriza um comportamento suspeito, e, por isso, também foram excluídas. Não foram observados dados ausentes.

Com isso, 197 respostas foram consideradas válidas para o desenvolvimento da análise dos dados, e, portanto, esse número alcançado atendeu satisfatoriamente os critérios estatísticos estabelecidos na literatura, utilizando-se como referência a regra definida por Hair, Hult, Ringle e Sarstedt (2014), que estabelece que o tamanho da mesma deve ser dez vezes maior ou igual ao número de indicadores do construto que possuir a maior quantidade de indicadores do modelo de mensuração, ou o tamanho da amostra deve ser dez vezes maior ou igual ao número da maior quantidade de caminhos direcionados para um determinado construto do modelo estrutural.

\section{ANÁLISE E DISCUSSÃO DOS RESULTADOS}

\subsection{Caracterização da amostra}

Os aspectos demográficos da amostra são apresentados quanto à idade e ao sexo dos respondentes. Os números mostram que 40,1\% dos entrevistados tinham entre 21 e 30 anos, seguido por $35,5 \%$ entre as idades de 31 e 40 anos, e depois 14,7\% entre as idades de 41 e 50 . As faixas etárias localizadas nos extremos tiveram uma menor participação, representando $6,1 \%$ para os respondentes com idade acima de 51 anos, e 3,6\% para os jovens abaixo de 20 anos. Quanto ao sexo, 112 respondentes eram do feminino, enquanto 85 do masculino, caracterizando uma participação de 56,9\% de mulheres e 41,1\% de homens.

Do total de resposta válidas, quando os participantes foram perguntados sobre a frequência de uso de sites de compras online, 37,6\% responderam que utilizam mais de uma vez por mês, 35\% uma vez por mês, e $26,9 \%$ informaram que muito raramente realizam compras online. Analisando a frequência que os respondentes usam ou leem avaliações online de clientes antes de reservar um hotel, nota-se que dentre as respostas válidas, 25,9\% informaram que frequentemente se utilizam destas informações, e 58,4\% responderam que sempre usam ou leem avaliações online antes de reservarem uma hospedagem. Quanto a estimativa do número de vezes que os respondentes usaram ou leram avaliações online de clientes ao escolher um hotel ou pousada nos últimos 12 meses, 55,3\% disseram entre 1 a 3 vezes, 17,3\% entre 4 a 6 vezes, e $27,4 \%$ afirmaram que usaram as avaliações online mais de 7 vezes nesse período. Dessa forma, fica evidenciado que os respondentes apresentaram um perfil bastante qualificado para participarem da pesquisa.

\subsection{Critérios para processamento dos dados}

Para processamento dos dados no algoritmo PLS-SEM foram utilizados os critérios recomendados pela literatura quanto a tolerância crítica e ao número máximo de iterações, estabelecidos em 1e-07 e 300, respectivamente. Conforme Hair et al. (2014), se o algoritmo PLS-SEM não atingir a convergência em até 300 iterações, o mesmo não pode encontrar uma solução estável. Constatou-se que na presente pesquisa a convergência ocorreu na terceira iteração, portanto, a interrupção do processo se deu em função do atingimento da diferença mínima da soma dos pesos obtidos entre duas iterações, conforme estabelecido na tolerância crítica, e não pelo número máximo de iterações definido. 0 teste de Boostrapping foi realizado considerando o total de 5000 subamostras analisadas, conforme recomendado por Hair et al. (2014). Para todos os testes estatísticos utilizou-se o nível de significância $(\alpha)$ de 0,05.

\subsection{Avaliação do modelo de mensuração}

O modelo de mensuração no presente estudo é composto por indicadores reflexivos, e este foi submetido a testes para avaliação da confiabilidade e validade do modelo, especificamente quanto às análises de 
unidimensionalidade e confiabilidade interna dos indicadores, validade convergente e validade discriminante. Para análise da consistência interna, utilizou-se como referência o alfa de Cronbach (C. alfa), que segundo Hair et al. (2014) fornece uma estimativa da confiabilidade com base nas intercorrelações observadas entre os indicadores, tendo como faixa aceitável valores entre 0,60 e 0,90. Outra métrica usada para avaliar a unidimensionalidade de um modelo reflexivo é o coeficiente de DillonGoldstein's rho (DG. rho), que tem foco na variância da soma dos indicadores no construto de interesse (Sanchez, 2013). Segundo este autor, como regra geral, um construto é considerado unidimensional quando o DG. rho é maior que 0,7 , e este índice é considerado melhor do que o alfa de Cronbach porque leva em consideração até que ponto o construto explica seu bloco de indicadores. Observando os resultados da Tabela 1, todos os construtos apresentaram consistência interna e unidimensionalidade adequadas.

\begin{tabular}{lll}
\multicolumn{3}{l}{ Tabela 1- Unidimensionalidade } \\
\hline Construto & C.alpha & DG.rho \\
\hline QL & 0,70 & 0,83 \\
QN & 0,60 & 0,79 \\
NI & 0,83 & 0,89 \\
Cl & 0,82 & 0,88 \\
PU & 0,74 & 0,88 \\
IC & 0,89 & 0,94 \\
\hline
\end{tabular}

Fonte: Elaborado pelos autores

A análise da validade convergente foi feita considerando as cargas externas dos indicadores e a variância média extraída (AVE) relacionada aos construtos. De acordo com Hair et al. (2014), as cargas externas devem ser superiores a 0,708 e o valor de AVE, fator que explica a comunalidade dos indicadores do construto, deve ser maior que 0,5 .

Conforme apresentado na Tabela 2, os indicadores Q1, Q2, e Q3 apresentaram valores de cargas externas inferiores a 0,708, e AVE de 0,49 para o construto QL. Ao desconsiderar do modelo os indicadores Q1 e Q2, escolhidos por apresentarem as menores cargas externas do construto QL, o valor de AVE aumentou para 0,63. De acordo com Hair et al. (2014), quando há uma variação considerável na variância média extraída do construto, recomenda-se a retirada dos indicadores. Com a exclusão de Q1 e Q2, a carga externa do indicador Q3 alcançou 0,813, atendendo o valor crítico estabelecido na literatura.

Em relação ao indicador $\mathrm{Q} 7$, cuja carga externa foi de 0,66, a retirada do mesmo do modelo estrutural não alterou substancialmente o valor de AVE, e por isso, optou-se por mantê-lo no modelo de mensuração, conforme recomendado por Hair et al. (2014). Portanto, os resultados apresentados a seguir foram obtidos considerando a exclusão dos indicadores Q1 e Q2.

\begin{tabular}{|c|c|c|c|c|c|}
\hline Construto & Indicador & Cargas externas & & AVE & \\
\hline & Q1 & 0,642 & * & & \\
\hline & Q2 & 0,666 & * & & \\
\hline \multirow[t]{4}{*}{ QL } & Q3 & 0,671 & 0,813 & 0,49 & 0,63 \\
\hline & Q4 & 0,765 & 0,862 & & \\
\hline & Q5 & 0,734 & 0,692 & & \\
\hline & Q6 & 0,791 & 0,791 & & \\
\hline \multirow[t]{3}{*}{ QN } & Q7 & 0,661 & 0,661 & 0,54 & 0,54 \\
\hline & Q8 & 0,749 & 0,748 & & \\
\hline & Q9 & 0,783 & 0,783 & & \\
\hline \multirow{3}{*}{$\mathrm{NI}$} & Q10 & 0,853 & 0,853 & & \\
\hline & Q11 & 0,858 & 0,858 & 0,66 & 0,66 \\
\hline & Q12 & 0,763 & 0,763 & & \\
\hline \multirow{4}{*}{$\mathrm{Cl}$} & Q13 & 0,818 & 0,818 & & \\
\hline & Q14 & 0,735 & 0,735 & 0,65 & 0,65 \\
\hline & Q15 & 0,846 & 0,846 & & \\
\hline & Q16 & 0,814 & 0,814 & & \\
\hline \multirow{3}{*}{ PU } & Q17 & 0,895 & 0,894 & & \\
\hline & Q18 & 0,884 & 0,885 & 0,19 & 0,19 \\
\hline & Q19 & 0,813 & 0,813 & & \\
\hline \multirow{3}{*}{ IC } & Q20 & 0,922 & 0,922 & & \\
\hline & Q21 & 0,887 & 0,887 & 0,15 & 0,75 \\
\hline & Q22 & 0,831 & 0,831 & & \\
\hline
\end{tabular}

Fonte: Elaborado pelos autores 
A validade discriminante, que avalia se um construto é realmente diferente de outros num modelo empírico, foi analisada com base nas cargas cruzadas dos indicadores, e no comparativo entre as raízes quadradas dos valores de AVE dos construtos. Segundo Hair et al. (2014), a validade discriminante é atendida quando as cargas de um indicador de um construto forem maiores que as cargas deste indicador em relação aos outros construtos do modelo. De acordo com os resultados apresentados na Tabela 3, observa-se que a validade discriminante foi alcançada nesse estudo.

\begin{tabular}{cccccccc}
\multicolumn{2}{c}{ Tabela 3 - Validade discriminante - cargas cruzadas } \\
\hline \multirow{6}{c}{ Construto } & Indicador & QL & QN & NI & Cl & PU & IC \\
\multirow{2}{*}{ QL } & Q3 & 0,813 & 0,069 & 0,150 & 0,314 & 0,318 & 0,203 \\
& Q4 & 0,862 & 0,071 & 0,217 & 0,436 & 0,337 & 0,247 \\
& Q5 & 0,692 & 0,167 & 0,295 & 0,531 & 0,303 & 0,324 \\
QN & Q6 & 0,078 & 0,791 & 0,158 & 0,225 & 0,221 & 0,248 \\
& Q7 & 0,033 & 0,661 & 0,133 & 0,207 & 0,134 & 0,173 \\
& Q8 & 0,141 & 0,748 & 0,304 & 0,312 & 0,263 & 0,342 \\
NI & Q9 & 0,279 & 0,239 & 0,783 & 0,293 & 0,419 & 0,500 \\
& Q10 & 0,224 & 0,203 & 0,853 & 0,337 & 0,463 & 0,604 \\
& Q11 & 0,259 & 0,261 & 0,858 & 0,344 & 0,478 & 0,684 \\
& Q12 & 0,131 & 0,240 & 0,763 & 0,371 & 0,378 & 0,626 \\
Cl & Q13 & 0,365 & 0,335 & 0,423 & 0,818 & 0,504 & 0,502 \\
& Q14 & 0,340 & 0,311 & 0,247 & 0,735 & 0,299 & 0,389 \\
& Q15 & 0,494 & 0,269 & 0,352 & 0,846 & 0,473 & 0,445 \\
\multirow{4}{*}{ PU } & Q16 & 0,524 & 0,205 & 0,263 & 0,814 & 0,410 & 0,387 \\
& Q17 & 0,326 & 0,230 & 0,506 & 0,423 & 0,894 & 0,619 \\
& Q18 & 0,393 & 0,297 & 0,446 & 0,537 & 0,885 & 0,536 \\
& Q19 & 0,335 & 0,191 & 0,662 & 0,423 & 0,672 & 0,813 \\
IC & Q20 & 0,323 & 0,315 & 0,692 & 0,477 & 0,524 & 0,922 \\
& Q21 & 0,191 & 0,387 & 0,627 & 0,466 & 0,515 & 0,887 \\
& Q22 & 0,246 & 0,404 & 0,554 & 0,508 & 0,488 & 0,831 \\
\hline
\end{tabular}

Fonte: Elaborado pelos autores

Adicionalmente, a validade discriminante foi analisada conforme o critério estabelecido por Fornell-Larcker, sendo esta uma segunda e mais conservadora abordagem (Hair et al., 2014). Nesta análise, cada uma das raízes quadradas de AVE deve ser maior do que os outros coeficientes de correlação para uma validade discriminante adequada, critério plenamente atendido, conforme resultados apresentados na Tabela 4.

\begin{tabular}{|c|c|c|c|c|c|c|}
\hline Construto & QL & QN & $\mathrm{NI}$ & $\mathrm{Cl}$ & PU & IC \\
\hline QL & 0,792 & & & & & \\
\hline QN & 0,127 & 0,736 & & & & \\
\hline $\mathrm{NI}$ & 0,277 & 0,288 & 0,815 & & & \\
\hline $\mathrm{Cl}$ & 0,537 & 0,346 & 0,411 & 0,804 & & \\
\hline PU & 0,404 & 0,295 & 0,536 & 0,538 & 0,890 & \\
\hline IC & 0,324 & 0,364 & 0,741 & 0,541 & 0,650 & 0,864 \\
\hline
\end{tabular}

\subsection{Avaliação do modelo estrutural}

A análise do modelo estrutural tem como objetivo identificar o alinhamento entre os conceitos teóricos e os modelos de caminho obtidos a partir das observações empíricas, visando reconhecer se os pressupostos teóricos serão confirmados estatisticamente (Hair et al., 2014). Nessa seção, os índices avaliados foram a significância e relevância dos coeficientes de caminho $(\beta)$, e os coeficientes de determinação $\left(R^{2}\right)$, que serão apresentados e discutidos a seguir.

Preliminarmente, o teste de colinearidade entre os construtos foi realizado com o objetivo de avaliar se os mesmos podem ser redundantes em função de possuírem alta correlação. Os resultados obtidos apresentados na Tabela 5 não demonstraram tal efeito indesejado, pois os fatores de inflação da variância (VIF) dos construtos foram inferiores a cinco, considerando o critério de avaliação estabelecido por Hair et al., 2014. 


\begin{tabular}{|c|c|c|c|c|}
\hline Construto & $\mathrm{QL}$ & QN & $\mathrm{NI}$ & $\mathrm{Cl}$ \\
\hline PU & 1,425 & 1,180 & 1,249 & 1,694 \\
\hline
\end{tabular}

Fonte: Elaborado pelos autores

Com base na análise dos resultados, os coeficientes de caminho observados nas relações entre os construtos que compõem o modelo estrutural apresentaram-se em sua maioria significativos, visto que os $p$-valores foram todos menores que o nível de significância de 0,05 , com exceção da hipótese $\mathrm{H} 2$, conforme apresentado na Tabela 6.

Tabela 6 - Coeficientes de caminhos entre os construtos e análise de significância $(\alpha=0,05)$

\begin{tabular}{ccccc}
\hline Relação & Coeficiente $(\boldsymbol{\beta})$ & Erro Padrão & P valor & Hipótese \\
\hline QL -> PU & 0,139 & 0,065 & 0,0346 & H1 - confirmada \\
QN -> PU & 0,074 & 0,059 & 0,2154 & H2 - não confirmada \\
NI -> PU & 0,356 & 0,061 & 0,0000 & H3 - confirmada \\
Cl -> PU & 0,292 & 0,071 & 0,0001 & H4 - confirmada \\
PU -> Cl & 0,650 & 0,054 & 0,0000 & H5 - confirmada \\
\hline
\end{tabular}

Fonte: Elaborado pelos autores

Mais especificamente, as hipóteses $\mathrm{H} 1, \mathrm{H} 3$ e H4 foram confirmadas, pois as relações entre os construtos qualidade da informação, necessidade da informação, e credibilidade da informação com a percepção de utilidade da informação foram significativas, apresentando coeficientes de caminho de 0,139, 0,356 e 0,292, respectivamente. Dessa forma, comparando os valores dos coeficientes, pode-se observar que a necessidade da informação é a variável que mais impacta a percepção de utilidade da informação, seguido da credibilidade da informação, e da qualidade da informação.

Esses resultados, em termos de significância dos coeficientes de caminho entre os construtos QL, $\mathrm{NI}$ e $\mathrm{Cl}$ com PU, estão em linha com os resultados encontrados por Erkan e Evans (2016), conforme apresentado: $\mathrm{QL}->\mathrm{PU}(\beta=0,26, p<0,05)$; $\mathrm{NI} \rightarrow \mathrm{PU}(\beta=0,41, p<0,05) ;$ e $\mathrm{Cl}->\mathrm{PU} \quad(\beta=0,22, p<0,05)$. Em relação à relevância, observa-se que no presente estudo a necessidade da informação apresentou a maior relevância, quando comparado aos outros construtos antecessores da percepção da utilidade da informação, resultado também constatado no estudo de Erkan e Evans (2016). No entanto, quanto aos construtos qualidade da informação e credibilidade da informação, verifica-se uma inversão em termos da relevância. Os resultados encontrados nesta pesquisa demonstram que a credibilidade da informação exerce maior influência na percepção de utilidade da informação, em comparação ao construto qualidade da informação. Esse resultado evidencia diferenças em termos de percepções dos consumidores brasileiros quanto às avaliações online disponibilizadas em sites e plataformas digitais, quando comparado aos resultados obtidos junto a consumidores do Reino Unido, unidade de análise utilizada no estudo de Erkan e Evans (2016). Essa diferença evidencia que aspectos culturais e socioeconômicos podem estar influenciando a percepção de utilidade da informação, devido aos diferentes contextos que os consumidores desses dois países estão inseridos.

Observando os achados do estudo de Bataineh (2015), a relação entre o construto QN com IC mostrou-se significativa, evidenciando que essa variável influencia a percepção das avaliações online e o processo de compra, porém com menor relevância quando comparados aos outros dois construtos avaliados (qualidade e credibilidade da informação). Como verificado na Tabela 6, a hipótese H2, que sustentava que a quantidade de informações afeta positivamente a percepção de utilidade, não foi confirmada. Esse resultado mostra que para o grupo de informantes que participou da presente pesquisa, a quantidade de avaliações online a respeito de uma hospedagem não se caracteriza como um fator que influencie decisivamente a percepção de utilidade da informação, analisando esse resultado comparativamente aos demais construtos antecessores. Esse achado reforça que a percepção de utilidade da informação é muito mais influenciada pela necessidade da informação, pela sua credibilidade e qualidade, devendo serem estes os principais fatores a orientarem a gestão de informações de plataformas digitais de reservas de serviços de hospedagem.

A hipótese $\mathrm{H} 5$, que sustentava uma relação de influência positiva entre a percepção de utilidade da informação e a intenção de compra, foi confirmada, apresentando um coeficiente de caminho significativo 
de 0,65. Dessa forma, fica evidente que a percepção dos consumidores a respeito da utilidade das avalições online disponibilizadas nos sites de hospedagem influencia fortemente o processo de decisão de compra deste tipo de serviço.

Os coeficientes de determinação obtidos para os construtos endógenos do modelo são apresentados na Tabela 7. Observou-se em relação ao construto $\mathrm{PU}$ um valor de $\mathrm{R}^{2}$ igual a 0,426 , indicando que $42,6 \%$ da variação observada em PU é explicada pelos construtos QL, QN, NI e Cl. Em relação a IC, verificou-se um valor de $\mathrm{R}^{2}$ de 0,423 , indicando que a variação deste construto é explicada em $42,3 \%$ por PU.

Tabela 7 - Valores de $\mathrm{R}^{2}$ dos construtos endógenos

\begin{tabular}{l|l}
\hline Construto & $\mathbf{R}^{2}$ \\
\hline PU & 0,429 \\
IC & 0,423 \\
\hline
\end{tabular}

Fonte: Elaborado pelos autores

Portanto, conforme objetivo central desta pesquisa, este resultado demonstra que a percepção de utilidade das avaliações online influencia positivamente o processo de intenção de compra de serviços de hospedagem. A Figura 2 apresenta a síntese dos resultados do modelo estrutural.

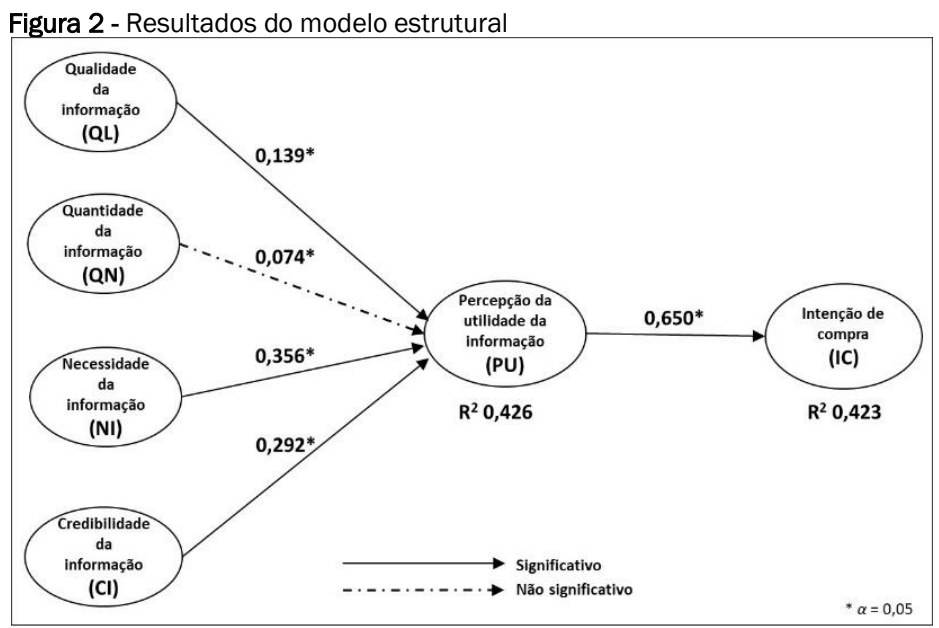

Fonte: Elaborado pelos autores

\section{CONCLUSÕES}

O presente estudo foi desenvolvido empiricamente por meio da coleta de opiniões de usuários familiarizados com o uso de avaliações online para análise das opções de serviços de hotelaria, e se propôs a investigar a influência da percepção de utilidade das avaliações online na intenção de compra de serviços de hotelaria, bem como avaliar as variáveis que impactam a percepção de utilidade das avaliações online. Os resultados encontrados demonstram que os construtos qualidade da informação, necessidade da informação e credibilidade da informação influenciam positivamente a percepção de utilidade, e por isso, é cada vez mais importante que os gestores de marketing e de operações de negócios tenham um maior entendimento sobre esse processo.

Verificou-se que em termos de relevância, a necessidade de informação é o fator que mais afeta a percepção de utilidade dos consumidores em relação as avaliações online. Ou seja, em um ambiente de troca de informação digital, com grande volume de dados, as opiniões disponíveis em sites especializados de hospedagem são cada vez mais úteis para os consumidores, que têm uma crescente necessidade de conhecer referências dos serviços ofertados, e, por isso, buscam as avaliações online para pautarem as suas decisões de compra. Diante dessa verificação, é recomendado que os gestores de marketing e de relacionamento com consumidores de sites de avaliação e de reserva de hospedagens desenvolvam plataformas de comunicação claras e de fácil acesso, favorecendo, assim, o processo de busca de informações dos consumidores. 
O segundo fator que mais influencia a percepção de utilidade das avalições online é a credibilidade da informação, sendo este mais relevante quando comparado à qualidade da informação disponibilizada nos sites de hotelaria. A ordem desse resultado difere em relação aos achados da pesquisa de Erkan e Evans (2016), realizada com usuários do Reino Unido. Como provável causa dessa inversão, pode-se atribuir os diferentes contextos, cultural e socioeconômico, observados entre esses países. Esse ponto nos faz refletir se o consumidor brasileiro apresenta um comportamento mais cético em relação às opiniões online, quando comparado a consumidores de países desenvolvidos. Outro fator que pode ter influenciado a diferença neste resultado é o foco que as pesquisas tiveram. 0 estudo de Erkan e Evans (2016) teve como objetivo analisar a influência das mídias sociais na intenção de compra, dentro de um contexto amplo, sem especificar o produto ou serviço. Nesta pesquisa, o foco foi analisar a influência das avalições online nas decisões de compras de serviços de hotelaria, que apresentam um alto grau de subjetividade, por sua natureza mais intangivel, além de serem muito susceptíveis a fatores socioculturais. Esse fato pode ter contribuído para uma maior relevância do construto credibilidade da informação.

Ademais, como principal contribuição teórica deste estudo, destaca-se a avaliação do modelo proposto por Erkan e Evans (2016) num contexto associado ao mercado de hotelaria, validando para esse segmento de mercado que a percepção de utilidade das avaliações online influencia positivamente o processo de compra de serviços de hospedagem. Dessa forma, os resultados encontrados ampliam a base de conhecimento no campo do marketing digital associado ao mercado de hotelaria.

Como contribuição prática, reconhecendo a importância da credibilidade das informações disponíveis nas plataformas digitais, é recomendado que os sites online invistam em ferramentas, e em processos de certificação que minimizem a produção e a circulação de conteúdos que não representem com veracidade a opinião dos usuários. É importante também comunicar adequadamente para os consumidores esse controle quanto à credibilidade das informações, de maneira que os mesmos se sintam mais seguros no uso das avaliações online no momento de realizarem suas compras. Ainda de acordo com os resultados encontrados, a adoção de medidas gerenciais no sentido de melhorar a qualidade da informação disponível nos sites e mídias sociais é outro ponto que merece atenção por parte dos profissionais que gerenciam o marketing digital nas organizações que estão inseridas nesse ambiente. Plataformas digitais que possibilitam a inserção e a difusão de avaliações online relevantes, compreensíveis, com informações suficientes e objetivas, são melhores percebidas pelos consumidores potenciais, e têm maior capacidade de influenciar positivamente o processo de reserva de hospedagens.

Por fim, conclui-se que cada vez mais, em função da crescente interconectividade digital, facilitada pela expansão dos sites especializados e das mídias sociais, as pessoas decidem as suas compras de serviços de hospedagem influenciadas pela percepção de utilidade das avaliações online. Pode-se dizer também, a partir dos achados da pesquisa, que indiretamente, as decisões de compras dos consumidores são afetadas pela necessidade que os mesmos têm de buscar informações sobre as opções de hotelaria, pela credibilidade em relação às avaliações disponibilizadas, e pela qualidade das informações online. Quanto a quantidade de avaliações disponibilizadas nas plataformas digitais, os resultados da pesquisa mostraram que esse fator não afeta significativamente a percepção de utilidade da informação de consumidores brasileiros, e, por consequência, também não influencia a intenção de compra.

Sugere-se como pesquisa futura uma maior investigação sobre a credibilidade da informação, procurando identificar a influência de aspectos cognitivos e afetivos relacionados a esse construto na intenção de compra. Dessa forma, questões como a reputação das plataformas digitais, e a caracterização dos perfis dos consumidores que postam avaliações online, podem ser investigados para identificar os seus efeitos na credibilidade da informação e na intenção de compra de serviços de hospedagem. Pesquisas recentes têm identificado a credibilidade da informação como mediadora de relações entre construtos de caráter informativo e comportamental, como por exemplo, as desenvolvidas por Forgas-Coll, Palau-Saumell, Sánchez-García e Caplliure-Giner (2014), Namahoot e Laohavichien (2018), e Frederico, Teixeira, Ahmed e Ghani (2017), sendo esta uma outra oportunidade de pesquisa futura relacionada ao processo de escolha de hospedagem. Além disso, a identificação de eventuais efeitos moderadores nesse processo, como por exemplo, em termos de sexo, idade e renda dos consumidores também pode ser objeto de novas pesquisas. 
Esta pesquisa, como outros estudos, também apresenta limitações. A amostra, por mais que tenha sido composta por respondentes com perfil adequado, foi coletada em uma única região metropolitana brasileira, com grupos de pessoas ligadas a instituições de ensino superior, limitando a generalização dos resultados em função de particularidades regionais que possam afetar a análise do efeito das avaliações online na intenção de compra de serviços de hotelaria. Outra limitação é o fato desta pesquisa ter abordado a influência das avaliações online disponibilizadas em plataformas digitais de maneira ampla, sem se concentrar em um site ou rede social específica, que poderia gerar resultados e conclusões diferentes.

\section{REFERÊNCIAS}

Almeida, G. S. de. (2018). Qualidade do serviço dos meios de hospedagem capixabas e a satisfação do consumidor segundo as avaliações do site Tripadvisor. Universidade Federal do Espírito Santo.

Bataineh, A. Q. (2015). The Impact of Perceived e-WOM on Purchase Intention: The Mediating Role of Corporate Image. International Journal of Marketing Studies, 7(1), 126-137. https://doi.org/10.5539/ijms.v7n1p126

Bhattacherjee, A., \& Sanford, C. (2006). Influence Processes for Information Technology Acceptance: an Elaboration Likelihood Model. MIS Quarterly, 30(4), 805-825.

Browning, V., So, K. K. F., \& Sparks, B. (2013). The Influence of Online Reviews on Consumers' Attributions of Service Quality and Control for Service Standards in Hotels. Journal of Travel \& Tourism Marketing, 30(1-2), 23-40. https://doi.org/10.1080/10548408.2013.750971

Cheung, C. M. K., Lee, M. K. O., \& Rabjohn, N. (2008). The impact of electronic word-of-mouth: The adoption of online opinions in online customer communities. Internet Research, 18(3), 229-247. https://doi.org/10.1108/10662240810883290

Cheung, C. M. K., \& Thadani, D. R. (2012). The impact of electronic word-of-mouth communication: A literature analysis and integrative model. Decision Support Systems, 54(1), 461-470. https://doi.org/10.1016/j.dss.2012.06.008

Davis, F. (1989). Perceived usefulness, perceived ease of use, and user acceptance of information technology. MIS Quarterly, 13(3), 319-340. https://doi.org/10.1016/S0305-0483(98)00028-0

Elseidi, R. I., \& El-Baz, D. (2016). Electronic word of mouth effects on consumers' brand attitudes, brand image and purchase intention: an empirical study in Egypt. The Business and Management Review, 7(5), 268-276.

Erkan, I., \& Evans, C. (2016). The influence of eWOM in social media on consumers' purchase intentions: An extended approach to information adoption. Computers in Human Behavior, 61, 47-55. https://doi.org/10.1016/i.chb.2016.03.003

Farley, A., \& Murched, N. (2016). How Culture Moderates the Effect of Trust on online Shopping Frequency. Malardalen University.

Forgas-Coll, S., Palau-Saumell, R., Sánchez-García, J., \& María Caplliure-Giner, E. (2014). The role of trust in cruise passenger behavioral intentions: The moderating effects of the cruise line brand. Management Decision, 52(8), 1346-1367. https://doi.org/10.1108/MD-09-2012-0674

Frederico, E., Teixeira, N. C., Ahmed, S., \& Ghani, A. (2017). Determinantes da lealdade aos sites de compras coletivas (SCCs). REGE - Revista de Gestão, 24, 281-290.

https://doi.org/https://doi.org/10.1016/j.rege.2016.08.004

Hair, J. F., Hult, G. T. M., Ringle, C. M., \& Sarstedt, M. (2014). Partial Least Squares Structural Equation Modeling. Handbook of Market Research (1a). California: Sage Publications. https://doi.org/10.1007/9783-319-05542-8_15-1

Hajli, M. N. (2013). A study of the impact of social media on consumers. International Journal of Market Research, 56(January), 387-404. https://doi.org/10.2501/U M R-2014-025

Hernández-Méndez, J., Muñoz-Leiva, F., \& Sánchez-Fernández, J. (2015). The influence of e-word-of-mouth on travel decision-making: consumer profiles. Current Issues in Tourism, 18(11), 1001-1021. https://doi.org/10.1080/13683500.2013.802764

IBGE. (2016). Pesquisa de Serviço de hospedagem. Rio de Janeiro. 
Jalilvand, M. R., \& Samiei, N. (2012). The effect of electronic word of mouth on brand image and purchase intention. An empirical study in the automobile. Marketing Intelligence\&Planning, 30(4), 460-476. https://doi.org/10.1108/02634501211231946

Jardim, G. D. S., \& Sant'Anna, A. L. P. (2007). Turismo on-line: oportunidades e desafios em um novo cenário profissional. Revista Acadêmica Observatório de Inovação Do Turismo, 2(3), 01. https://doi.org/10.12660/oit.v2n3.5671

Ladhari, R., \& Michaud, M. (2015). EWOM effects on hotel booking intentions, attitudes, trust, and website perceptions. International Journal of Hospitality Management, 46, 36-45. https://doi.org/10.1016/j.ijhm.2015.01.010

Lee, E. J., \& Shin, S. Y. (2014). When do consumers buy online product reviews? Effects of review quality, product type, and reviewer's photo. Computers in Human Behavior, 31(1), 356-366. https://doi.org/10.1016/j.chb.2013.10.050

Lee, J., Park, D. H., \& Han, I. (2008). The effect of negative online consumer reviews on product attitude: An information processing view. Electronic Commerce Research and Applications, 7(3), 341-352. https://doi.org/10.1016/j.elerap.2007.05.004

Lee, K. T., \& Koo, D. M. (2015). Evaluating right versus just evaluating online consumer reviews. Computers in Human Behavior, 45, 316-327. https://doi.org/10.1016/j.chb.2014.12.036

Medeiros, S. A., Gosling, M., \& Vera, L. A. R. (2015). Emoções em Experiências Negativas de Turismo : um estudo sobre a influência na insatisfação. Revista Turismo Em Análise, 26(1), 188-215. https://doi.org/http://dx.doi.org/10.11606

Namahoot, K. S., \& Laohavichien, T. (2018). Assessing the intentions to use internet banking. International Journal of Bank Marketing, 36(2), 256-276. https://doi.org/10.1108/IJBM-11-2016-0159

Ong, B. S. (2012). The Perceived Influence of User Reviews in the Hospitality Industry. Journal of Hospitality Marketing and Management, 21(5), 463-485. https://doi.org/10.1080/19368623.2012.626743

Park, D.-H., Lee, J., \& Han, I. (2007). The Effect of On-Line Consumer Reviews on Consumer Purchasing Intention: The Moderating Role of Involvement. International Journal of Electronic Commerce, 11(4), 125148. https://doi.org/10.2753/JEC1086-4415110405

Park, H., Xiang, Z., Josiam, B., \& Kim, H. (2014). Personal profile information as cues of credibility in online travel reviews. Anatolia, 25(1), 13-23. https://doi.org/10.1080/13032917.2013.820203

Phocuswright.Com. (2016). Retrieved March 14, 2019, from https://www.phocuswright.com/TravelResearch/Market-Overview-Sizing/Latin-America-Online-Travel-Overview-Third-Edition

Phocuswright.Com. (2018). Retrieved May 5, 2018, from https://www.phocuswright.com/TravelResearch/Technology-Innovation/The-Brazilian-Digital-Traveler

Sanchez, G. (2013). Pls path modeling with r. Berkeley: Trowchez Editions.

Silva, G. L. da, Mendes Filho, L., \& Marques Júnior, S. (2019). Análise da Percepção dos Consumidores de Meios de Hospedagem em Relação ao Uso das Online Travel Agencies (OTAs). Revista Brasileira de Pesquisa Em Turismo, 13(1), 40-57. https://doi.org/10.7784/rbtur.v13i1.1468

Statista.Com. (2018). Retrieved July 25, 2018, from https://www.statista.com/

Sussman, S. W., \& Siegal, W. S. (2003). Informational In uence in Organizations: An Integrated Approach to Knowledge Adoption. Information Systems Research, 14(1), 47-65. https://doi.org/10.1287/isre.14.1.47.14767

Tsao, W. C., Hsieh, M. T., Shih, L. W., \& Lin, T. M. Y. (2015). Compliance with eWOM: the influence of hotel reviews on booking intention from the perspective of consumer conformity. International Journal of Hospitality Management, 46, 99-111. https://doi.org/10.1016/j.ijhm.2015.01.008

Wani, T. A., Ali, S. W., \& Farooq, T. (2016). Determinants of online purchase intentions: a study of indian buyers. Amity Journal of Management Research AJMR Amity Journal of Management Research, 1(11), 94109. Retrieved from http://amity.edu/UserFiles/admaa/233Paper 7.pdf

Wolny, J., \& Mueller, C. (2013). Analysis of Fashion Consumers' Motives to Engage in Electronic Word-ofMouth Communication through Social Media. Journal of Marketing Management, 29(5-6), 562-583. https://doi.org/http://dx.doi.org/10.1080/0267257X.2013.778324 


\section{Informações dos autores}

\section{Rinaldo de Castro Oliveira}

Mestrando em Administração de Empresas pela Universidade Federal do Espírito Santo - UFES da Linha de Pesquisa em Estratégia, Inovação e Desempenho Organizacional. Realiza pesquisas na área de Administração, com ênfase na Gestão de Operações, Inovação e Tecnologia.

Contribuição: definição da concepção da pesquisa, contemplando a questão problema e o modelo conceitual, elaboração da revisão da literatura e do instrumento de coleta dos dados, e condução da análise dos resultados e conclusões. E-mail: rinaldo@dmep.com.br ORCID - https://orcid.org/0000-0002-3724-0388

\section{Elaine Christina Gonçálves Dá Rós Baldam}

Mestranda em Administração de Empresas pela Universidade Federal do Espírito Santo - UFES da Linha de Pesquisa em Estratégia, Inovação e Desempenho Organizacional.

Pós-graduada em Engenharia de Produção - IFES/ES

Contribuição: elaboração da revisão da literatura, condução da coleta de dados e da análise estatística dos dados, e contribuição na análise dos resultados e conclusões.

E-mail: elaine.daros.baldam@gmail.com

ORCID - https://orcid.org/0000-0002-3557-670X

\section{Fábio Reis da Costa}

Mestre em Administração pela Universidade Federal do Espírito Santo - UFES.

Professor da Fundação de Apoio à Escola Técnica do Estado do Rio de Janeiro - FAETEC;

Administrador da Universidade Estadual do Norte Fluminense Darcy Ribeiro - UENF.

Contribuição: revisão da introdução, objetivos e da justificativa do tema de pesquisa, bem como do referencial teórico, validação do modelo conceitual e hipóteses, e revisão da análise dos resultados e discussões.

E-mail: reis.frc@gmail.com

ORCID - https://orcid.org/0000-0003-0507-0668

\section{Anderson Soncini Pelissari}

Doutor em Engenharia de Produção. Professor do Programa de Pós-graduação em Administração - Mestrado e Doutorado - PPGADM/UFES. Pesquisador da Linha de Pesquisa - Estratégia, Inovação e Desempenho Organizacional - Núcleo de Estudos em Tecnologias e Processos Organizacionais - TecPrO. Realiza pesquisas nas áreas de Estratégias de Marketing e de Negócios.

Contribuição: revisão da introdução, objetivos e da justificativa do tema de pesquisa, bem como do referencial teórico, e validação do modelo conceitual e hipóteses. Revisão geral do artigo, com ênfase na análise dos resultados e na identificação de contribuições teóricas e práticas.

E-mail: asoncinipelissari@gmail.com

ORCID - https://orcid.org/0000-0003-1567-8159 
APÊNDICE A - Indicadores e escala de mensuração

Pergunta chave para os As informações sobre os serviços de hotelaria que são compartilhados nas mídias construtos QL, QN, NI, Cl e sociais, sites especializados...

PU

Qualidade da Informação

Q1 - Eu acho que as avaliações online são bem fundamentadas.

(QL)

Q2 - Eu acho que as avaliações online são objetivas.

(Park, Lee e Han, 2007)

Q3 - Eu acho que as avaliações online são de fácil compreensão.

Q4 - Eu acho que as avaliações online são claras.

Q5 - Em geral, acho que a qualidade das avaliações online é alta.

Q6 - O número elevado de avaliações online fornecidos por usuários de hotéis leva a

Quantidade da Informação entender que o hotel é frequentado.

(QN)

Q7 - A quantidade elevada de avaliações online fornecidas por usuários de hotéis leva a entender que o hotel está na moda.

(Bataineh, 2015)

Q8 - Hotéis bem ranqueados e com grande número de recomendações online leva a entender que o hotel oferece serviços de alta qualidade.

Q9 - Eu gosto de usar as avaliações online quando considero reservar um hotel novo para mim.

Necessidade de Informação $(\mathrm{NI})$

(Chu e Kim, 2011)

Q10 - Se eu tiver pouca experiência sobre o hotel, geralmente eu utilizo as avaliações online.

Q11 - Eu costumo consultar as avaliações online para escolher a melhor alternativa de hospedagem.

Q12 - Frequentemente eu analiso um conjunto de avaliações online antes de fazer uma reserva de hotel.

Credibilidade da informação

(Cl)

Q13 - Eu acho que as avaliações online são convincentes.

Q14 - Eu acho que as avaliações online são robustas.

(Prendergast et al., 2010)

Q15 - Eu acho que as avaliações online são confiáveis.

Q16 - Eu acho que as avaliações online são precisas.

Percepção da utilidade da informação (PU)

(Bailey e Pearson, 1983)

Intenção de compra (IC)

(Coyle and Thorson, 2001;

Prendergast et al., 2010)

Q17- Eu acho que as avaliações online são geralmente úteis.

Q18 - Eu acho que as avaliações online são geralmente informativas.

Depois de considerar as informações sobre os serviços de hospedagem que são compartilhados nas mídias sociais e sites especializados ...

Q19 - É muito provável que eu reserve um hotel considerando as avaliações online.

Q20 - Reservarei um hotel na próxima vez que eu precisar de uma hospedagem le-

vando em consideração avaliações online.

Q21 - Eu vou definitivamente experimentar um hotel levando em consideração avaliações online.

Q22 - Eu vou recomendar um hotel para meus amigos levando em consideração avaliações online. 